\title{
A novel analytical technique to obtain the solitary solutions for nonlinear evolution equation of fractional order
}

\author{
Abdul Ghaffar ${ }^{1,2}$, Ayyaz Ali3 , Sarfaraz Ahmed ${ }^{4}$, Saima Akram5 ${ }^{5}$ Moin-ud-Din Junjua ${ }^{3}$, \\ Dumitru Baleanu ${ }^{6,7,8}$ and Kottakkaran Sooppy Nisar ${ }^{9 *}$ (D)
}

"Correspondence:

n.sooppy@psau.edu.sa; ksnisar1@gmail.com

${ }^{9}$ Department of Mathematics, College of Arts and Science, Prince Sattam bin Abdulaziz University, Wadi Aldawaser, Saudi Arabia Full list of author information is available at the end of the article

\section{Springer}

\begin{abstract}
We investigate some solitary wave results of time fractional evolution equations. By employing the extended rational $\exp \left(\left(-\frac{\psi^{\prime}}{\psi}\right)(\eta)\right)$-expansion method, a few different results including kink, singular-kink, multiple soliton, and periodic wave solutions are formally generated. It is worth mentioning that the solutions obtained are more general with more parameters. The exact solutions are constructed in the form of exponential, trigonometric, rational, and hyperbolic functions. With the choice of proper values of parameters, graphs to some of the obtained solutions are drawn. On comparing some special cases, our solutions are in good agreement with the results published previously and the remaining are new.

MSC: 35R11;26A33; 74J35; 35C08

Keywords: Simplified modified Camassa-Holm (SMCH) equation; Fractional calculus; Caputo's derivative of fractional order; Solitary wave solutions; Extended rational $\exp \left(\left(\frac{\psi^{\prime}}{\psi}\right)(\eta)\right)$-expansion method
\end{abstract}

\section{Introduction}

In the exceptional development of nonlinear sciences and engineering, during the last few decades, many researchers seem to be interested in obtaining exact and numerical solutions for nonlinear partial differential (NLPD) equations. One used the modified variational iteration algorithm-II [1], the direct algebraic method [2], the extended trial equation method [3], the enhanced $\left(\frac{G^{\prime}}{G}\right)$-expansion method [4], the GERFM method [5, 6] using generalized fractional integral conditions [7], applying the non-compact measure and Monch's theorem [8-10], the modified simple equation [11], the function transformation method [12], the extended modified mapping method [13], the function transformation method [14] and the reductive perturbation method [15]. The study of exact solutions of nonlinear evolution equations plays a major role to explore the internal mechanism of nonlinear phenomena $[3,13]$. Fractional calculus is a dominant tool in several nonlinear fields such as plasma physics, fluid mechanics, solid-state physics, optical fibers, quantum field theory, biophysics, chemical kinematics, electricity, chemistry, biology, geochemistry,

(c) The Author(s) 2020. This article is licensed under a Creative Commons Attribution 4.0 International License, which permits use sharing, adaptation, distribution and reproduction in any medium or format, as long as you give appropriate credit to the original author(s) and the source, provide a link to the Creative Commons licence, and indicate if changes were made. The images or other third party material in this article are included in the article's Creative Commons licence, unless indicated otherwise in a credit line to the material. If material is not included in the article's Creative Commons licence and your intended use is not permitted by statutory regulation or exceeds the permitted use, you will need to obtain permission directly from the copyright holder. To view a copy of this licence, visit http://creativecommons.org/licenses/by/4.0/. 
propagation of shallow water waves and engineering $[7,10,16]$. For this purpose many techniques were used such as the homogeneous balance method [17], the exp-function method [18], the improved extended F-expansion method [19], and the homotopy perturbation method [20]. The Camassa-Holm (CH) equation is

$$
u_{t}+2 k u_{x}-u_{x x t}+\beta u u_{x}=2 u_{x} u_{x x}+u u_{x x x} .
$$

The above equation gained the fame as a model describing the unidirectional propagation of shallow water waves over a flat bottom [21] and in this perception, if $\beta$ is positive, the solitary solutions are smooth solitons. In Eq. (1) $k$ and $\beta$ are some parameters, $u(x, t)$ is the dependent variable, while $x$ and $t$ are the spatial and temporal variables. It is also considered as an equation with a bi-Hamiltonian structure, particularly, when $\beta=0$, the above stated equation has a peakon type solution. So, the $\mathrm{CH}$ equation has some other peakon solutions and smooth solutions. Song and Tian investigated the modified Camassa-Holm (MCHE) equation in [22],

$$
u_{t}+2 k u_{x}-u_{x x t}+\beta u^{n} u_{x}=2 u_{x} u_{x x}+u u_{x x x} .
$$

Further, one assumed that the soliton changes with $\eta=x-V t$, where $V$ is the speed of wave propagation and $t$ is the temporal variable. For $V>0$, the wave moves in the positive $\mathrm{x}$-direction, whereas for $V<0$, the wave moves in the negative $\mathrm{x}$-direction, then the terms $2 u_{x} u_{x x}+u u_{x x x}$ of Eq. (2) will be very small and the above equation becomes

$$
u_{t}+2 k u_{x}-u_{x x t}+\beta u u_{x}=0
$$

Consider Eq. (3), the modified Camassa-Holm (MCH) equation [22], which is known as the simplified modified Camassa-Holm $(\mathrm{SMCH})$ equation

$$
u_{t}+2 k u_{x}-u_{x x t}+\beta u^{n} u_{x}=0 .
$$

In this paper, we consider $n=2$,

$$
u_{t}+2 k u_{x}-u_{x x t}+\beta u^{2} u_{x}=0
$$

With the help of He's semi-inverse method [23] we have obtained the analytical solutions of the $\mathrm{CH}$ equation and the $\mathrm{SMCH}$ equation, Abbasbandy via the homotopy method [24] obtained traveling wave solutions of the SMCH equation. Recently many solitary solutions of SMCH equation were obtained $[25,26]$. Here, we utilize the extended rational $\exp \left(\left(-\frac{\psi^{\prime}}{\psi}\right)(\eta)\right)$-expansion method for solving the simplified modified Camassa-Holm $(\mathrm{SMCH})$ equation.

\section{Description of method}

We will explain the extended rational $\exp \left(\left(-\frac{\psi^{\prime}}{\psi}\right)(\eta)\right)$-expansion method for establishing solitary solutions. Suppose the fractional PDE is

$$
\psi\left(u, D_{t}^{\dot{a}} u, D_{t}^{2 \dot{a}} u, D_{t}^{\dot{a}} u_{x}, u_{x}, u_{x x}, \ldots\right)=0, \quad 0 \leq \alpha \leq 1, t>0,
$$


where $D_{t}^{\alpha} u, D_{x}^{\alpha} u, D_{x x}^{\alpha} u$, are fractional derivatives, $u$ is any unknown function and $\psi$ is a polynomial involving $\mathrm{u}$ and its different derivatives. So as to solve (6), the steps are as follows.

Step I: We assume the following equation:

$$
\eta=x \pm V \frac{t^{\alpha}}{\Gamma(\alpha+1)}
$$

where $V$ is the speed of the wave propagation, $u=u(\eta)$.

Equations (6) and (7) yield the ODE

$$
\psi\left(u, \pm V u^{\prime}, k u^{\prime}, k^{2} u^{\prime \prime}, V^{2} u^{\prime \prime}, \ldots\right)=0 .
$$

Step II: Suppose the solution of Eq. (8) is expressed as

$$
\begin{aligned}
& u(\eta)=\frac{a[0]+\frac{d}{d \eta}\left(\sum_{n=1}^{M}\left(a_{n}\left(e^{\psi(\eta)}\right)^{n}\right)\right)}{\sum_{n=0}^{M}\left(b_{n}\left(e^{\psi(\eta)}\right)^{n}\right)}, \\
& \psi^{\prime}(\eta)=\mu \exp (\psi(\eta))+\exp (-\psi(\eta))+\lambda,
\end{aligned}
$$

while, $a_{n}, b_{n}$ are arbitrary constants and $\psi(\eta), a_{n} \neq 0, b_{n} \neq 0, \psi(\eta)$ satisfies Eq. (1), we got solutions from Eq. (10) as follows.

Family I: When $\lambda^{2}-4 \mu>0$,

$$
\psi(\eta)=\ln \left\{\left(-\sqrt{\left(\lambda^{2}-4 \mu\right)} \operatorname{Tanh}\left((\eta+c) \frac{\sqrt{\left(\lambda^{2}-4 \mu\right)}}{2}\right)-\lambda\right) \frac{1}{2 \mu}\right\} .
$$

Family II: When $-4 \mu+\lambda^{2}<0$,

$$
\psi(\eta)=\ln \left\{\left(\sqrt{\left(-4 \mu+\lambda^{2}\right)} \operatorname{Tan}\left((\eta+c) \frac{\sqrt{\left(\lambda^{2}-4 \mu\right)}}{2}\right)-\lambda\right) \frac{1}{2 \mu}\right\}
$$

Family III: When $\lambda \neq 0, \mu=0$ and $\lambda^{2}-4 \mu>0$,

$$
\psi(\eta)=-\ln \left\{\frac{\lambda}{e^{\left(\lambda\left(\eta+k_{1}\right)\right)}-1}\right\} .
$$

Family IV: When $\mu \neq 0, \lambda \neq 0$ and $\lambda^{2}-4 \mu=0$,

$$
\psi(\eta)=\ln \left\{\frac{\left(2 \lambda\left(\eta+k_{1}\right)+4\right)}{\left(\lambda^{2}\left(\eta+k_{1}\right)\right)}\right\} .
$$

Family V: When $\mu=0, \lambda^{2}-4 \mu=0$ and $\lambda=0$,

$$
\varphi(\eta)=\ln \left(\eta+k_{1}\right)
$$

Step III: Exploring the value of $M$ from Eq. (8). From Eq. (8) and Eq. (10), we have a polynomial of $e^{M \varphi(\eta)}$. By comparing, we get a system of equations with $V, \lambda, \mu, a_{n}$, and putting in Eq. (9) all cases of Eq. (10), we obtain traveling wave results of Eq. (6). 


\section{Caputo's fractional derivative}

Definition 3.1 ([27]) A real function, $h(x), x>0$, is supposed to be in space $C_{\alpha}$ if there exists any real number $p(>\alpha)$, such that

$$
h(x)=x^{p} h_{1}(x), \quad \text { where } h_{1}(x) \in C[0, \infty) .
$$

Definition $3.2([27])$ A real function $h(x), x>0$, is assumed to be in space if any real number $C_{\alpha}^{m}, m \in \mathbb{N} \cup\{0\}$, if $h^{(m)} \in C_{\alpha}$.

Definition 3.3 ([27]) Let $h \in C_{\alpha}$ and $\alpha \geq-1$, then for the Riemann-Liouville (R-L) (leftsided) integral of left-sided order $\mu, \mu>0$, we get

$$
I_{t}^{\mu} h(x, t)=\frac{1}{\Gamma(\mu)} \int_{0}^{t}(t-T)^{\mu-1} h(x, T) d T, \quad t>0,
$$

where $\Gamma(\mu)$ is the Gamma function.

Definition 3.4 ([27]) The Caputo fractional derivative of the left-sided function $h(x)$ with respect to $t, h \in C_{-1}^{m}, m \in \mathbb{N} \cup\{0\}$, is given as

$$
\begin{aligned}
& D_{t}^{\mu} h(x, t)=\frac{\partial^{m}}{\partial t^{m}} h(x, t), \quad \mu=m \\
& =I_{t}^{m-\mu} \frac{\partial^{m}}{\partial t^{m}} h(x, t), \quad m-1 \leq \mu<m, m \in \mathbb{N}, \\
& I_{t}^{\mu} D_{t}^{\mu} h(x, t)=h(x, t)-\sum_{k=0}^{m-1} \frac{\partial^{k} h}{\partial t^{k}}(x, 0) \frac{t^{k}}{k !}, \quad m-1<\mu \leq m, m \in \mathbb{N}, \\
& I_{t}^{\mu} t^{\nu}=\frac{\Gamma(v+1)}{\Gamma(\mu+v+1)} t^{\mu+\nu} .
\end{aligned}
$$

\section{The simplified modified Camassa-Holm (SMCH) equation}

Consider the simplified modified Camassa-Holm (SMCH) equation

$$
D_{t}^{\alpha} u+2 k u_{x}-u_{x x t}+\beta u^{2} u_{x}=0
$$

where $k \in \Re$ and $\beta>0$ are parameters. We obtain

$$
\eta=x-V \frac{t^{\alpha}}{\Gamma(\alpha+1)}, \quad u(x, t)=u(\eta)
$$

we can convert Eq. (11) into an ODE,

$$
(2 k-V) u-V u^{\prime \prime}+\frac{1}{3} \beta u^{3}+P=0,
$$

therefore the trial solution of suggested algorithm is

$$
u(\eta)=\frac{\frac{d}{d \eta}\left(\sum_{n=1}^{M}\left(a_{n}\left(e^{Y(\eta)}\right)^{n}\right)\right)+a[0]}{\sum_{n=0}^{M}\left(b_{n}\left(e^{Y(\eta)}\right)^{n}\right)} .
$$


From (10), (12) and (13), we get

$$
H_{0}+H_{1} e^{\varphi(\eta)}+H_{2} e^{2 \varphi(\eta)}+H_{3} e^{3 \varphi(\eta)}+H_{4} e^{4 \varphi(\eta)}+H_{5} e^{5 \varphi(\eta)}+H_{6} e^{6 \varphi(\eta)}=0,
$$

where $H_{1}, H_{2}, H_{3}, H_{4}, H_{5}, H_{6}$ are given as follows:

$$
\begin{aligned}
& H_{1}=3 V \lambda^{3} a_{1} b_{0}{ }^{2}-3 V \lambda^{2} a_{0} b_{0} b_{1}-12 V \lambda^{2} a_{1} b_{0} b_{1}, \\
& H_{2}=-3 V \lambda^{3} a_{1} b_{0} b_{1}+21 V \lambda^{2} \mu a_{1} b_{0}{ }^{2}+3 V \lambda^{2} a_{0} b_{1}{ }^{2}, \\
& H_{3}=6 V \lambda^{2} \mu a_{1} b_{0} b_{1}+36 V \mu^{2} \lambda a_{1} b_{0}{ }^{2}+3 V \lambda^{2} \mu a_{0} b_{1}{ }^{2}, \\
& H_{4}=3 V \lambda^{2} \mu a_{1} b_{1}{ }^{2}+27 V \mu^{2} \lambda a_{1} b_{0} b_{1}+18 V \mu^{3} \mu a_{1} b_{0}{ }^{2}, \\
& H_{5}=9 V \mu^{2} \lambda a_{1} b_{1}{ }^{2}+18 V \mu^{3} a_{1} b_{0} b_{1}+3 \beta \mu^{2} \lambda a_{1}{ }^{3}, \\
& H_{6}=6 V \mu^{3} a_{1} b_{1}{ }^{2}+\beta \mu^{3} a_{1}{ }^{3} .
\end{aligned}
$$

We obtained the coefficients of algebraic equations:

$$
\left[H_{0}=0, H_{1}=0, H_{2}=0, H_{3}=0, H_{4}=0, H_{5}=0, H_{6}=0\right] .
$$

Solving these algebraic equations by using Maple, we obtain the solution sets, and by choosing some specific cases we get the following.

\section{Solution 1}

$$
\begin{array}{ll}
a_{0}=\frac{a_{1}\left(\lambda b_{0}-b_{1}\right)}{b_{1}}, & P=-\frac{\lambda a_{1}\left(-\beta \lambda^{2} a_{1}^{2}+3 V b_{1}{ }^{2}-6 k b_{1}{ }^{2}\right)}{3 b_{1}{ }^{3}}, \\
\lambda=\lambda, \quad b_{0}=b_{0}, & b_{1}=b_{1}, \quad a_{1}=a_{1}, \quad \beta=\beta, \quad \mu=0 .
\end{array}
$$

Case III: Whenever $\mu=0$ and $\lambda \neq 0$, and $\lambda^{2}-4 \mu>0$,

$$
u_{1}(\eta)=\frac{a_{1}\left(\lambda b_{0}-b_{1}\right) \lambda}{b_{1}\left(b_{1} e^{-\lambda\left(\frac{V t^{\alpha}}{\Gamma(\alpha+1)}-x\right)}+\lambda b_{0}-b_{1}\right)} .
$$

Case IV: When, $\lambda \neq 0, \lambda^{2}-4 \mu=0$ and $\mu \neq 0$,

$$
u_{2}(\eta)=\frac{a_{1}\left(\lambda b_{0}-b_{1}\right) \lambda^{2}\left(\frac{V t^{\alpha}}{\Gamma(\alpha+1)}-x\right)}{b_{1}\left(\lambda^{2} t b_{0} \frac{V t^{\alpha}}{\Gamma(\alpha+1)}-2 \lambda b_{1} \frac{V t^{\alpha}}{\Gamma(\alpha+1)}-\lambda^{2} x b_{0}+2 \lambda x b_{1}+2 b_{1}\right)} .
$$

Case V: If $\mu=0$, and $\lambda=0$,

$$
u_{3}(\eta)=\frac{a_{1}\left(\lambda b_{0}-b_{1}\right)}{b_{1}\left(b_{1} \frac{V t^{\alpha}}{\Gamma(\alpha+1)}-x b_{1}-b_{0}\right)}
$$

\section{Solution 2}

$$
b_{1}=b_{1}, \quad a_{1}=0, \quad a_{0}=a_{0}, \quad P=0, \quad \beta=0,
$$




$$
\lambda=\frac{\sqrt{V(V-2 k)}}{V}, \quad b_{0}=\frac{b_{1} \sqrt{V(V-2 k)}}{V-2 k}, \quad \mu=0 .
$$

Case III: Whenever $\mu=0$ and $\lambda \neq 0$, and $\lambda^{2}-4 \mu>0$,

$$
u_{4}(\eta)=\frac{\sqrt{V(V-2 k)} a_{0} e^{\frac{\left(\frac{V t^{\alpha}}{\Gamma(\alpha+1)}-x\right) \sqrt{V(V-2 k)}}{V}}}{V b_{1}} .
$$

Case IV: When $\lambda \neq 0, \lambda^{2}-4 \mu=0$, and $\mu \neq 0$,

$$
u_{5}(\eta)=-\frac{a_{0}(V-2 k)\left(\frac{V t^{\alpha}}{\Gamma(\alpha+1)}-x\right)}{b_{1}\left(\sqrt{V(V-2 k)} \frac{V^{\alpha}}{\Gamma(\alpha+1)}-\sqrt{V(V-2 k)} x-2 V\right)} .
$$

Case V: While $\mu=0$, and $\lambda=0$,

$$
u_{6}(\eta)=-\frac{a_{0}(V-2 k)}{b_{1}\left(2 k \frac{V t^{\alpha}}{\Gamma(\alpha+1)}+V x-\frac{V^{2} t^{\alpha}}{\Gamma(\alpha+1)}-2 k x+\sqrt{V(V-2 k)}\right)} .
$$

\section{Solution 3}

$$
\begin{aligned}
& b_{1}=b_{1}, \quad a_{1}=0, \quad a_{0}=a_{0}, \quad b_{0}=-\frac{b_{1} \sqrt{V(V-2 k)}}{V-2 k}, \\
& P=0, \quad \lambda=-\frac{\sqrt{V(V-2 k)}}{V}, \quad \beta=0, \quad \mu=0 .
\end{aligned}
$$

Case III: Whenever $\mu=0$ and $\lambda \neq 0$, and $\lambda^{2}-4 \mu>0$,

$$
u_{7}(\eta)=-\frac{a_{0} e^{-\frac{\sqrt{V(V-2 k)}\left(\frac{V t^{\alpha}}{\Gamma(\alpha+1)}-x\right)}{V}}(\sqrt{V(V-2 k)})}{V b_{1}} .
$$

Case IV: When $\mu \neq 0$, and $\lambda \neq 0, \lambda^{2}-4 \mu=0$,

$$
u_{8}(\eta)=\frac{a_{0}(V-2 k)\left(\frac{V t^{\alpha}}{\Gamma(\alpha+1)}-x\right)}{b_{1}\left(\sqrt{V(V-2 k)} \frac{V t^{\alpha}}{\Gamma(\alpha+1)}-\sqrt{V(V-2 k)} x+2 V\right)} .
$$

Case V: When $\mu=0$, and $\lambda=0$,

$$
u_{9}(\eta)=-\frac{a_{0}(V-2 k)}{b_{1}\left(-V x+2 k x+\frac{V^{2} t^{\alpha}}{\Gamma(\alpha+1)}+\sqrt{V(V-2 k)}\right)-2 k \frac{V t^{\alpha}}{\Gamma(\alpha+1)}} .
$$

\section{Solution 4}

$$
\begin{aligned}
& b_{1}=b_{1}, \quad a_{1}=0, \quad a_{0}=a_{0}, \quad \lambda=\lambda, \quad b_{0}=\frac{2 \lambda b_{1} V}{V \lambda^{2}+2 V-4 k}, \\
& P=0, \quad \beta=-\frac{24 V\left(V^{2}-4 k V+4 k^{4}\right) b_{1}^{2}}{\left(V \lambda^{2}+2 V-4 k\right)^{2} a_{0}^{2}}, \quad \mu=\frac{V \lambda^{2}+2 V-4 k}{4 V} .
\end{aligned}
$$

Case II: Whenever $\mu \neq 0$, and $\lambda^{2}-4 \mu<0$,

$$
u_{10}(\eta)=-\frac{a_{0}\left(V \lambda^{2}+2 V-4 k\right) \sqrt{2}}{4 b_{1} V \tan \left(\frac{1}{2}\left(\frac{V t^{\alpha}}{\Gamma(\alpha+1)}-x\right) \sqrt{2} \sqrt{\frac{V-2 k}{V}} \sqrt{\frac{V-2 k}{V}}\right)} .
$$


Case III: While $\lambda \neq 0$, and $\mu=0$,

$$
u_{11}(\eta)=-\frac{\left(V \lambda^{2}+2 V-4 k\right) \lambda a_{0}}{b_{1}\left(e^{-\lambda\left(\frac{V \alpha^{\alpha}}{\Gamma(\alpha+1)}-x\right)} V \lambda^{2}+V \lambda^{2}+2 e^{-\lambda\left(\frac{V t^{\alpha}}{\Gamma(\alpha+1)}-x\right)} V-4 e^{-\lambda\left(\frac{V \alpha^{\alpha}}{\Gamma(\alpha+1)}-x\right)} k-2 V+4 k\right)} .
$$

Case IV: When $\mu \neq 0, \lambda^{2}-4 \mu=0$, and $\lambda \neq 0$,

$$
u_{12}(\eta)=-\frac{a_{0}\left(V \lambda^{2}+2 V-4 k\right) \lambda^{2}\left(\frac{V t^{\alpha}}{\Gamma(\alpha+1)}-x\right)}{2 b_{1}\left(-V \lambda^{2}-2 \lambda V-4 \lambda k \frac{V t^{\alpha}}{\Gamma(\alpha+1)}-4 x k \lambda-2 V+4 k+2 \lambda^{2} \frac{V t^{\alpha}}{\Gamma(\alpha+1)}\right)} .
$$

Case V: When $\mu=0$, and $\lambda=0$,

$$
u_{13}(\eta)=-\frac{\left(V \lambda^{2}+2 V-4 k\right) a_{0}}{b_{1}\left(-x V \lambda^{2}-2 V \lambda-4 k \frac{V t^{\alpha}}{\Gamma(\alpha+1)}-2 V x+2 \lambda^{2} \frac{t^{\alpha}}{\Gamma(\alpha+1)}+4 k x+\lambda^{2} \frac{V^{2} t^{\alpha}}{\Gamma(\alpha+1)}\right)} .
$$

\section{Solution 5}

$$
\begin{aligned}
& b_{1}=\frac{b_{0}\left(V \lambda+\sqrt{\left.V^{2}-2 k V\right)}\right.}{2 V}, \quad P=-\frac{a_{0}\left(\lambda\left(V \lambda+\sqrt{V^{2}-2 k V}\right)-V \lambda^{2}-V+2 k\right)}{2 b_{0}}, \\
& \lambda=\lambda, \quad b_{0}=b_{0}, \quad \beta=0, \quad \mu=\frac{V \lambda^{2}-V+2 k}{4 V}, \quad a_{1}=0, \quad a_{0}=a_{0} .
\end{aligned}
$$

Case I: Whenever $\mu \neq 0$, and $\lambda^{2}-4 \mu>0$,

$$
\begin{aligned}
u_{14}(\eta)=-\frac{a_{0}\left(V \lambda^{2}-V+2 k\right)}{b_{0} \sqrt{\frac{V-2 k}{V}} \tan \left(\frac{1}{2}\left(\frac{-x+V t^{\alpha}}{\Gamma(\alpha+1)}\right) \sqrt{\frac{V-2 k}{V}}\right) V \lambda} \\
\left.\quad+\sqrt{V(V-2 k)} \sqrt{-\frac{V-2 k}{V}} \tan \left(\frac{1}{2} \sqrt{\frac{V-2 k}{V}}\left(\frac{-x+V t^{\alpha}}{\Gamma(\alpha+1)}\right)\right)-\lambda \sqrt{V(V-2 k)}\right)-V+2 k
\end{aligned}
$$

Case II: If $\mu \neq 0$, and $\lambda^{2}-4 \mu<0$,

$$
\begin{aligned}
u_{15}(\eta)=-\frac{a_{0}\left(V \lambda^{2}-V+2 k\right)}{b_{0} \sqrt{-\frac{V-2 k}{V}} \tan \left(\frac{1}{2}\left(\frac{-x+V t^{\alpha}}{\Gamma(\alpha+1)}\right) \sqrt{-\frac{V-2 k}{V}}\right) V \lambda} \\
\left.\quad+\sqrt{V(V-2 k)} \sqrt{-\frac{V-2 k}{V}} \tan \left(\frac{1}{2} \sqrt{-\frac{V-2 k}{V}}\left(\frac{-x+V t^{\alpha}}{\Gamma(\alpha+1)}\right)\right)+\lambda \sqrt{V(V-2 k)}\right)+V-2 k
\end{aligned}
$$

Case III: Whenever $\mu=0$ and $\lambda \neq 0$, and $\lambda^{2}-4 \mu>0$,

$$
u_{16}(\eta)=\frac{2 a_{0} V \lambda}{b_{1}\left(V \lambda e^{-\lambda\left(\frac{-x+V t^{\alpha}}{\Gamma(\alpha+1)}\right)}+\sqrt{V(V-2 k)} e^{-\lambda\left(\frac{-x+V^{\alpha}}{\Gamma(\alpha+1)}\right)}+V \lambda-\sqrt{V(V-2 k)}\right)} .
$$

Case IV: When $\mu \neq 0, \lambda \neq 0$, and $\lambda^{2}-4 \mu=0$,

$$
u_{17}(\eta)=-\frac{a_{0} V \lambda^{2}\left(-x+\frac{V t^{\alpha}}{\Gamma(\alpha+1)}\right)}{b_{0}\left(\sqrt{(V-2 k)} V \lambda \frac{V t^{\alpha}}{\Gamma(\alpha+1)}-\sqrt{V(V-2 k)} \lambda x-V \lambda-\sqrt{V(V-2 k)}\right)} .
$$

Case V: Whenever $\mu=0$, and $\lambda=0$,

$$
u_{18}(\eta)=-\frac{2 a_{0} V}{b_{0}\left(\lambda^{2} \frac{V t^{\alpha}}{\Gamma(\alpha+1)}+\sqrt{V(V-2 k)} \frac{V t^{\alpha}}{\Gamma(\alpha+1)}-V \lambda x-2 V-\sqrt{V(V-2 k)} x\right)} .
$$




\section{Solution 6}

$$
\begin{aligned}
& \lambda=\frac{\sqrt{2} \sqrt{V(2 V \mu-V+2 k)}}{V}, \quad b_{0}=\frac{\sqrt{2} b_{1} \sqrt{V(2 V \mu-V+2 k)}}{2 \mu V}, \\
& b_{1}=b_{1}, \quad a_{1}=a_{1}, \quad a_{0}=\frac{-(V-2 k)}{2 V \mu}, \quad P=0, \quad \beta=-\frac{6 V b_{1}{ }^{2}}{a_{1}{ }^{2}}, \quad \mu=\mu .
\end{aligned}
$$

Case I: If $\mu \neq 0$ and $\lambda^{2}-4 \mu>0$,

$$
u_{19}(\eta)=-\frac{(V-2 k) a_{1}}{V b_{1} \tanh \left(\frac{1}{2}\left(\frac{-x+V t^{\alpha}}{\Gamma(\alpha+1)}\right) \sqrt{-\frac{2 V-4 k}{V}} \sqrt{-\frac{2 V-4 k}{V}}\right)} .
$$

Case II: If $\mu \neq 0$ and $\lambda^{2}-4 \mu<0$,

$$
u_{20}(\eta)=\frac{(V-2 k) a_{1} \sqrt{2}}{2 V b_{1} \sqrt{\frac{V-2 k}{V}} \tan \left(\frac{1}{2}\left(\frac{-x+V t^{\alpha}}{\Gamma(\alpha+1)}-x\right) \sqrt{2} \sqrt{\frac{V-2 k}{V}}\right)} .
$$

\section{Analogy and analysis}

Several authors have been discussed the simplified modified Camassa-Holm (SMCH) equation by using different techniques for finding exact traveling wave results. Particularly, Liu et al. [22] used the $\left(G^{\prime} / G\right)$-expansion method, Najafi et al. [23], used He's semi-inverse method applied $\exp (-\varphi(\eta))$-expansion method and Redi et al. [19] applied an improved $\left(G^{\prime} / G\right)$-expansion method; Gundogdu et al. [28] applied the elliptic function expansion method to get the traveling wave solutions. Akber et al. [26] obtained solutions $u_{1}(\phi)$, $u_{3}(\phi), u_{6}(\phi), u_{7}(\phi)$ and $u_{8}(\phi)$ that are equivalent to our solutions $u_{10}(\eta), u_{19}(\eta)$ and $u_{20}(\eta)$. For instance, Akber et al. [26] obtained solutions $u_{1}(\phi)$ with suitable values of $m_{2}=0, d=0$, $B=0, \sqrt{\Omega}=1, A=1$ matching with our solution $u_{19}(\eta)$ (see Table 1 ). In the same manner Akber et al. [26] obtained solutions $u_{3}(\phi)$ with suitable values of $m_{2}=0, d=0, \sqrt{\Delta}=1$, $A=2$ matching with our obtained solution $u_{10}(\eta)$ (see Table 1). Mohyud-Din et al. [29] obtained solutions $u_{4}(\eta)$ and $u_{10}(\eta)$, for $k=1, b_{0}=\frac{1}{2}, \beta=-1, \eta=0, c_{1}=1$ that are equivalent to our obtained solution $u_{1}(\eta)$ for $\lambda=1, \mu=-1, k=1, a_{1}=2 \sqrt{3}, b_{0}=2, b_{1}=1, t=0$, $x=0$. Similarly for different values of Mohyud-Din et al. [29] obtained solutions $u_{2}(\eta)$, $u_{4}(\eta), u_{8}(\eta), u_{9}(\eta), u_{18}(\eta)$ and $u_{23}(\eta)$ that are equivalent to our solutions $u_{1}(\eta), u_{3}(\eta)$ and $u_{7}(\eta)$ (see Table 2). In Table 3, our solutions and those of Lu et al. [25] are compared. For instance, Lu et al. [25] addressed solution $u_{4}$ for the particular choices of $\beta_{1}=1, \sqrt{\beta_{1}}=5$, $\sqrt{\beta_{3}}=1 / 2, \epsilon=1, k=-1, \omega=1$ and $\xi_{0}=0$ is equivalent to our obtained solution $u_{19}(\eta)$ (see Table 3). The rest of the solutions are new. Hence, our method produces more solutions than other methods existing in the current literature. In our work all the results obtained have been verified through Maple to be found to be correct.

\section{Physical interpretation}

Now we provide graphical representations of some results with different parametric values. Figure 1 shows the periodic wave profile of $u_{1}(\eta)$ for $\alpha=0.2, a_{1}=11, \lambda=1, V=12$, $b_{0}=1, b_{1}=12$. Figure 2 represents the multiple soliton solution of $u_{3}(\eta)$ for $\alpha=0.25$, $a_{1}=11, V=12, b_{1}=11, \mu=3$, while Fig. 3 displays the profile of solitary wave of $u_{4}(\eta)$ for the choice of $\alpha=0.30, a_{1}=11, \lambda=1, V=12, b_{1}=11, \mu=3$. 
Table 1 Comparing the results of Akber et al. [26] and Liu et al. [22] with our results

\begin{tabular}{|c|c|}
\hline Obtained results & Results of Liu et al. and Akber et al. \\
\hline $\begin{array}{l}\text { If } m_{1}=\frac{(V-2 k) a_{1}}{V b_{1}} \sqrt{-\frac{2 V-4 k}{V}}=1 \text {, then the solution is } \\
u_{19}=m_{1} \operatorname{coth}\left(\frac{x-\frac{V t}{\Gamma(\alpha+1)}}{2}\right)\end{array}$ & $\begin{array}{l}\text { If } m_{2}=0, d=0, \sqrt{\Omega}=1, \Phi=x-\frac{v t}{\Gamma(\alpha+1)}, A=1 \text {, then the } \\
\text { solution is } u_{1}=m_{1} \operatorname{coth}\left(\frac{x-\frac{v t}{\Gamma(\alpha+1)}}{2}\right)\end{array}$ \\
\hline $\begin{array}{l}\text { If } m_{1}=\frac{(V-2 k) a_{1}}{V b_{1}} \sqrt{-\frac{2 V-4 k}{V}}=1 \text {, then the solution is } \\
u_{19}=m_{2} \operatorname{coth}\left(\frac{x-\frac{V t}{T(\alpha+1)}}{2}\right)\end{array}$ & $\begin{array}{l}\text { If } m_{2}=0, d=0, \sqrt{\Omega}=1, \Phi=x-\frac{v t}{\Gamma(\alpha+1)}, A=2 \text {, then the } \\
\text { solution is } u_{6}=m_{2} \operatorname{coth}\left(\frac{x-\frac{v t}{\Gamma(\alpha+1)}}{2}\right)\end{array}$ \\
\hline $\begin{array}{l}\text { If } \sqrt{-\frac{2 V-4 K}{V}}=\frac{1}{\sqrt{2}} \text { and } m_{1}=\frac{a_{0}\left(V \lambda^{2}+2 V+4 K\right)}{2 V b_{1}} \text {, then the } \\
\text { solution is } u_{10}=m_{1} \cot \left(\frac{\frac{V t}{\Gamma(\alpha+1)}-x}{2}\right)\end{array}$ & $\begin{array}{l}\text { If } m_{2}=0, d=0, B=0, \iota \sqrt{\Omega}=1, \Phi=\frac{v t}{T(\alpha+1)}-x, A=1, \\
\text { then the solution is } u_{3}=m_{1} \cot \left(\frac{\frac{V t}{\Gamma(\alpha+1)^{-x}}}{2}\right)\end{array}$ \\
\hline $\begin{array}{l}\text { If } \sqrt{-\frac{2 V-4 K}{V}}=\frac{1}{\sqrt{2}} \text { and } m_{2}=\frac{a_{0}\left(V \lambda^{2}+2 V+4 K\right)}{2 V b_{1}} \text {, then the } \\
\text { solution is } u_{10}=m_{2} \cot \left(\frac{\frac{V t}{\Gamma(\alpha+1)}-x}{2}\right)\end{array}$ & $\begin{array}{l}\text { If } m_{1}=\frac{1}{2}, d=0, \iota \sqrt{\Omega}=1, \Phi=\frac{v t}{\Gamma(\alpha+1)}-x, A=2 \text {, then } \\
\text { the solution is } u_{8}=m_{1} \cot \left(\frac{\frac{v t}{\Gamma(\alpha+1)}-x}{2}\right)\end{array}$ \\
\hline $\begin{array}{l}\text { If } \sqrt{-\frac{2 V-4 K}{V}}=1 \text { and } m_{2}=\frac{a_{1}(2 K+V)}{V b_{1}} \text { then the solution is } \\
u_{19}=m_{2} \operatorname{coth}\left(\frac{\frac{V t}{\Gamma(\alpha+1)^{-x}}}{2}\right)\end{array}$ & $\begin{array}{l}\text { If } m_{1}=\frac{1}{2}, d=0, \sqrt{\Delta}=1, \Phi=\frac{v t}{\Gamma(\alpha+1)}-x, A=2 \text {, then the } \\
\text { solution is } u_{7}=m_{2} \operatorname{coth}\left(\frac{\frac{V t}{2}(\alpha+1)^{-x}}{2}\right)\end{array}$ \\
\hline $\begin{array}{l}\text { If } \sqrt{-\frac{2 V-4 K}{V}}=1, a_{1}=1 \text { and } m_{2}=\frac{2 K+V}{V b_{1}}=\frac{2}{5} \text { then the } \\
\text { solution is } u_{20}=\frac{2}{5} \operatorname{coth}\left(\frac{V t}{\frac{\Gamma(\alpha+1)}{2}}\right)\end{array}$ & $\begin{array}{l}\text { If } m_{1}=\frac{1}{5}, m_{2}=0, d=0, \sqrt{\Delta}=1, \Phi=\frac{v_{t}}{\Gamma(\alpha+1)}-x, A=2 \text {, } \\
\text { then the solution is } u_{6}=\frac{2}{5} \operatorname{coth}\left(\frac{v_{t}(\alpha+1)}{2}\right)\end{array}$ \\
\hline $\begin{array}{l}\text { If } \sqrt{-\frac{2 V-4 K}{V}}=\frac{1}{\sqrt{2}} \text { and } m_{1}=\frac{a_{1}(-2 K+V)}{2 V b_{1}}=1 \text { then we get } \\
u_{20}=\cot \left(\frac{\frac{V t}{\Gamma(\alpha+1)}-x}{2}\right)\end{array}$ & 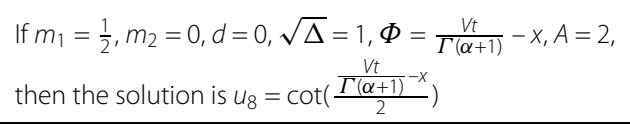 \\
\hline
\end{tabular}

Table 2 Comparing the results of Mohyud-Din et al. [17] and Najafi et al. [30] with our results

\begin{tabular}{|c|c|}
\hline Obtained results & Results of Najafi et al. and Mohyud-Din et al. \\
\hline $\begin{array}{l}\text { If } \lambda=1, \mu=-1, k=1, a_{1}=2 \sqrt{3}, b_{0}=2, b_{1}=1, t=0, \\
x=0 \text { then the solution is } u_{1}(\eta)=-2 \sqrt{3}\end{array}$ & $\begin{array}{l}\text { If } k=1, b_{1}=\frac{1}{2}, \beta=-1, \eta=0, c_{1}=1 \text { then the solution is } \\
u_{4}(\eta)=-2 \sqrt{3}\end{array}$ \\
\hline $\begin{array}{l}\text { If } \lambda=1, \mu=-1, k=1, a_{1}=2 \sqrt{3}, b_{0}=2, b_{1}=1, t=0, \\
x=0 \text { then the solution is } u_{1}(\eta)=-2 \sqrt{3}\end{array}$ & $\begin{array}{l}\text { If } k=1, b_{1}=\frac{1}{2}, \beta=-1, \eta=0, c_{1}=1 \text { then the solution is } \\
u_{10}(\eta)=-2 \sqrt{3}\end{array}$ \\
\hline $\begin{array}{l}\text { If } \lambda=\frac{2}{\sqrt{2}}, \mu=-1, k=1, a_{1}=3 \sqrt{6}, b_{0}=\sqrt{2}, b_{1}=1, \\
V=6 \sqrt{2}, t=1, x=0 \text { then the solution is } u_{3}(\eta)=\frac{3 \sqrt{6}}{5 \sqrt{2}}\end{array}$ & $\begin{array}{l}\text { If } \lambda=1, k=1, b_{0}=1, \beta=-1, \eta=0, c_{1}=1, \mu=1 \text { then the } \\
\text { solution is } u_{4}(\eta)=\frac{3 \sqrt{6}}{5 \sqrt{2}}\end{array}$ \\
\hline $\begin{array}{l}\text { If } \lambda=\frac{2}{\sqrt{2}}, \mu=-1, k=1, a_{1}=3 \sqrt{6}, b_{0}=\sqrt{2}, b_{1}=1 \\
V=6 \sqrt{2}, t=1, x=0 \text { then the solution is } u_{3}(\eta)=\frac{3 \sqrt{6}}{5 \sqrt{2}}\end{array}$ & $\begin{array}{l}\text { If } \lambda=1, k=1, b_{0}=1, \beta=-1, \eta=0, c_{1}=1, \mu=1 \text { then the } \\
\text { solution is } u_{9}(\eta)=\frac{3 \sqrt{6}}{5 \sqrt{2}}\end{array}$ \\
\hline $\begin{array}{l}\text { If } V=6, a_{1}=1, t=0, x=0, b_{1}=\frac{-1}{6}, k=\frac{5}{2} \text { then the } \\
\text { solution is } u_{7}(\eta)=\sqrt{6}\end{array}$ & $\begin{array}{l}\text { If } \lambda=\sqrt{-1}, k=1, b_{0}=0, \beta=1, k=1 \text { then the solution is } \\
u_{2}(\eta)=\sqrt{6}\end{array}$ \\
\hline \multirow[t]{2}{*}{$\begin{array}{l}\text { If } V=6, a_{1}=1, t=0, x=0, b_{1}=\frac{-1}{6}, k=\frac{5}{2} \text { then the } \\
\text { solution is } u_{7}(\eta)=\sqrt{6}\end{array}$} & $\begin{array}{l}\text { If } \lambda=\sqrt{-1}, k=1, b_{0}=0, \beta=1, k=1 \text { then the solution is } \\
u_{8}(\eta)=\sqrt{6}\end{array}$ \\
\hline & $\begin{array}{l}\text { If } k=-6, b_{1}=\frac{12}{\sqrt{6}}, \beta=-1, k=-6, c_{1}=\frac{\sqrt{6}}{12} \text { then the } \\
\text { solutions are } u_{18}(\eta)=u_{23}(\eta)=-2 \sqrt{3} \text {. It matches with } \\
\text { our obtained solution (i) in this table }\end{array}$ \\
\hline
\end{tabular}

Table 3 Comparing the results of Lu et al. [25] with our results

\begin{tabular}{|c|c|}
\hline Obtained results & Results of Lu et al. \\
\hline $\begin{array}{l}\text { If } H_{1}=\frac{a_{1}(V-2 k)}{b_{1} \sqrt{V(V-2 k)}}, a=0 \text { and } V=5 \text { then the solution is } \\
u_{19}=H_{1} \operatorname{coth}\left(\frac{5}{2}-x\right)\end{array}$ & $\begin{array}{l}\text { If } H_{1}=\frac{k \sqrt{6 \beta}}{\beta_{2} \sqrt{-\delta\left(\beta_{1} k^{2}+2\right)}}, \epsilon=1, k=-1, \omega=1, \beta_{1}=1, \\
\sqrt{\beta_{1}}=5, \sqrt{\beta_{3}}=\frac{1}{2} \text { and } \zeta_{0}=0 \text { then the solution is } \\
u_{4}=H_{1} \operatorname{coth}\left(\frac{5}{2}-x\right)\end{array}$ \\
\hline $\begin{array}{l}\text { If } H_{1}=\frac{a_{1}(V-2 k-)}{b_{1} \sqrt{V(V-2 k)}}, a=0 \text { and } V=5 \text { then the solution is } \\
u_{19}=H_{1} \operatorname{coth}\left(\frac{5}{2}-x\right)\end{array}$ & $\begin{array}{l}\text { If } H_{1}=\frac{2 \sqrt{3} k \beta_{1} \sqrt{\beta_{3} \beta}}{\beta_{2} \sqrt{-\delta\left(\beta_{1} k^{2}+2\right)}}, \epsilon=1, k=-1, \omega=1, \beta_{1}=1 \text {, } \\
\sqrt{\beta_{1}}=5, \sqrt{\beta_{3}}=\frac{1}{2} \text { and } \zeta_{0}=0 \text {, then the solution is } \\
u_{7}=H_{1} \operatorname{coth}\left(\frac{5}{2}-x\right)\end{array}$ \\
\hline
\end{tabular}




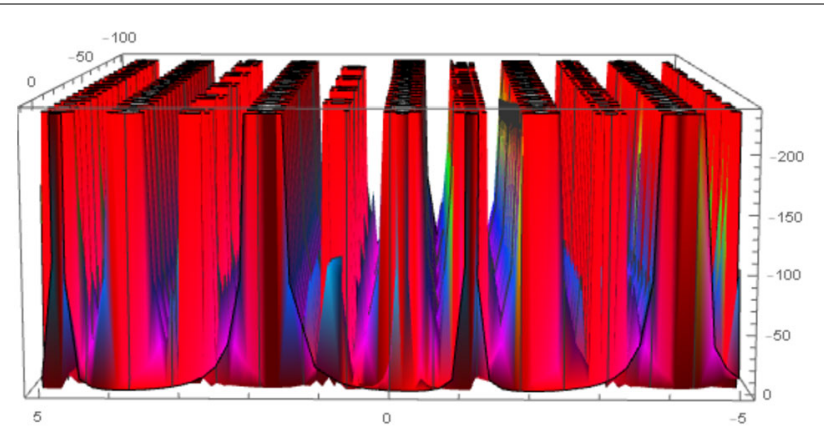

Figure 1 Periodic wave profile of $u_{1}(\eta)$ for $\alpha=0.2, a_{1}=11, \lambda=1, V=12, b_{0}=1, b_{1}=12$

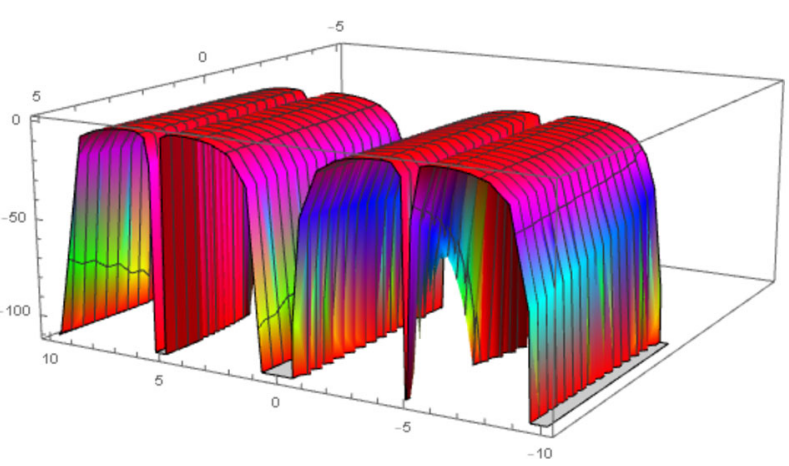

Figure 2 Multiple soliton solution of $u_{3}(\eta)$ for $\alpha=0.25, a_{1}=11, \lambda=1, V=12, b_{1}=11, \mu=3$

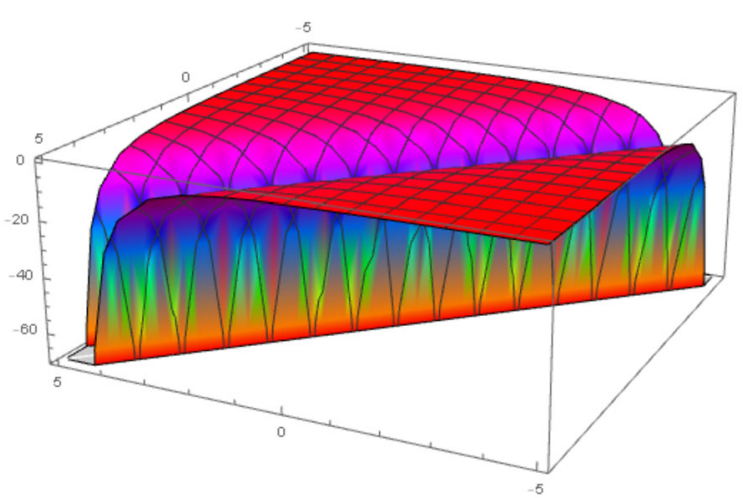

Figure 3 Solitary wave solution $u_{2}(\eta)$ for $\alpha=0.30, a_{1}=11, \lambda=1, V=12, b_{1}=11, \mu=3$

Again the solitary wave solution of $u_{4}(\eta)$ for $\alpha=0.75, a_{1}=1, \lambda=-1, V=0, b_{1}=1$ is obtained in Fig. 4. Figures 5 and 6 represent soliton solutions of $u_{4}(\eta)$ and $u_{14}(\eta)$ for different values of parameters. Solitons are special types of solitary waves that retain the identity upon interaction with other solitons and have implications in the field of cosmology of the universe. Figure 7 shows the kink wave solution of $u_{9}(\eta)$ for $\alpha=0.30, a_{1}=11, \lambda=0.77$, $b_{0}=1, V=10, b_{1}=100$. The kink waves are the solitary waves that are rise or descend from one asymptote state to another. Figure 8 displays the periodic solution of $u_{12}(\eta)$ for $\alpha=0.9, a_{1}=11, \lambda=-100, b_{0}=1, V=10$. The periodic solutions are traveling wave results 


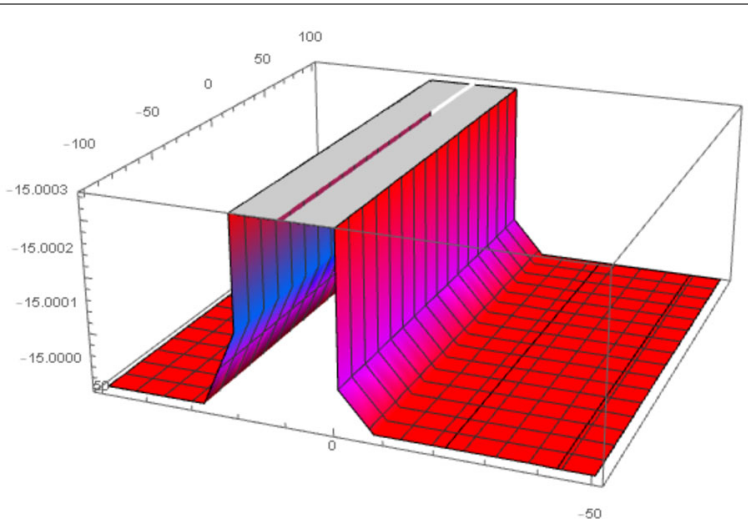

Figure 4 Solitary wave solution of $u_{4}(\eta)$ for $\alpha=0.75, a_{1}=1, \lambda=-1, V=0, b_{1}=1$

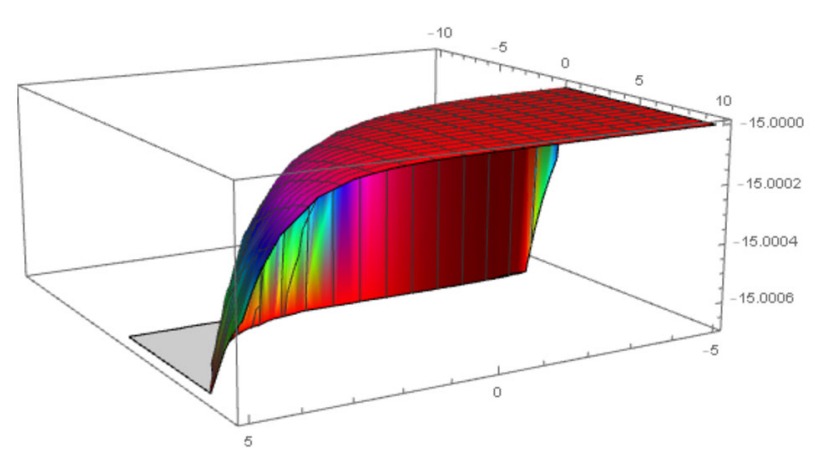

Figure 5 Soliton solution of $u_{6}(\eta)$ for $\alpha=0, a_{1}=1, \lambda=-1, V=0, b_{1}=1, k=3$

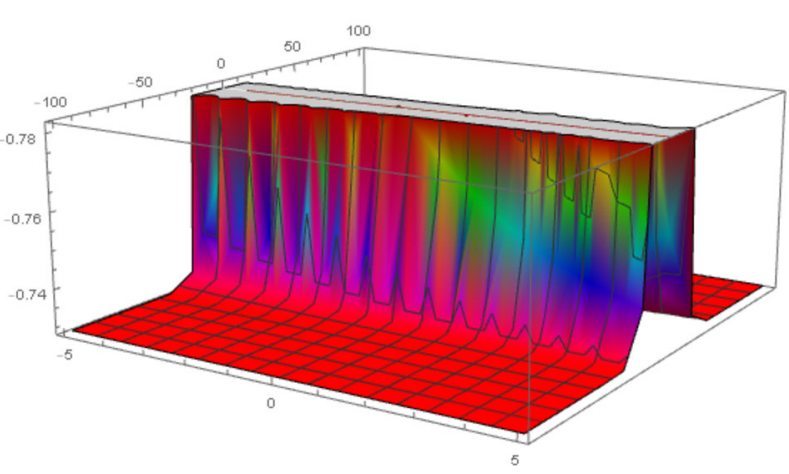

Figure 6 Solitonic solution of $u_{7}(\eta)$ for $\alpha=0.30, a_{1}=11, \lambda=0.77, b_{0}=1, b_{1}=100$

that are periodic. Finally, Fig. 9 represents the periodic solution of $u_{19}(\eta)$ for $\alpha=0.30$, $a_{1}=11, \lambda=0.77, b_{0}=1, V=10, b_{1}=100$.

Figure 10 shows the soliton solution of $u_{16}(\eta)$ for the choice of $\alpha=0.25, a_{0}=0.5, \lambda=-1$, $V=0.1, b_{0}=2, k=3$. Kink wave solution is obtained in Fig. 11 of $u_{14}(\eta)$ for $\alpha=0.9, a_{0}=$ $0.5, \lambda=-1, V=0.1, b_{0}=11, k=3$. Figure 12 displays the periodic solution of $u_{15}(\eta)$ for different values of $\alpha=0.1, a_{1} 1=11, \lambda=3, V=12, b_{0}=1, b_{1}=3$. Finally in Fig. 13 we have found solitonic solution of $u_{19}(\eta)$ for $\alpha=0.25, k=5, V=12, a_{1}=1, b_{1}=1$. 


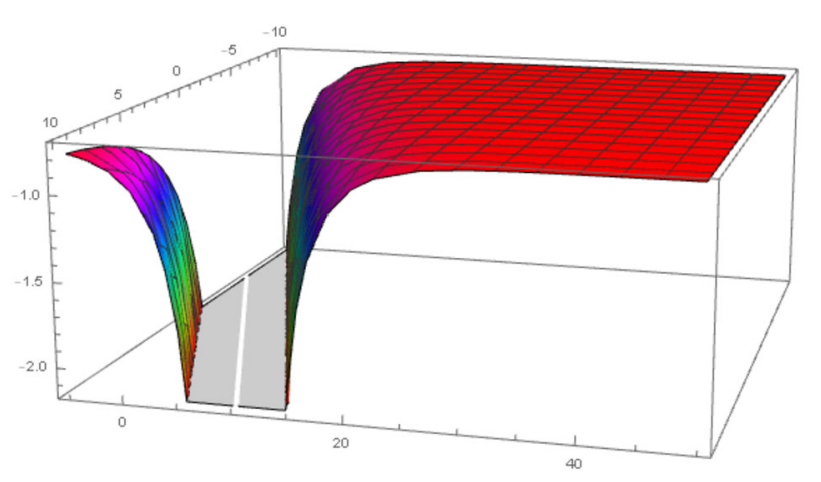

Figure 7 Kink wave of $u_{9}(\eta)$ for $\alpha=0.30, a_{1}=11, \lambda=0.77, b_{0}=1, V=10, b_{1}=100$

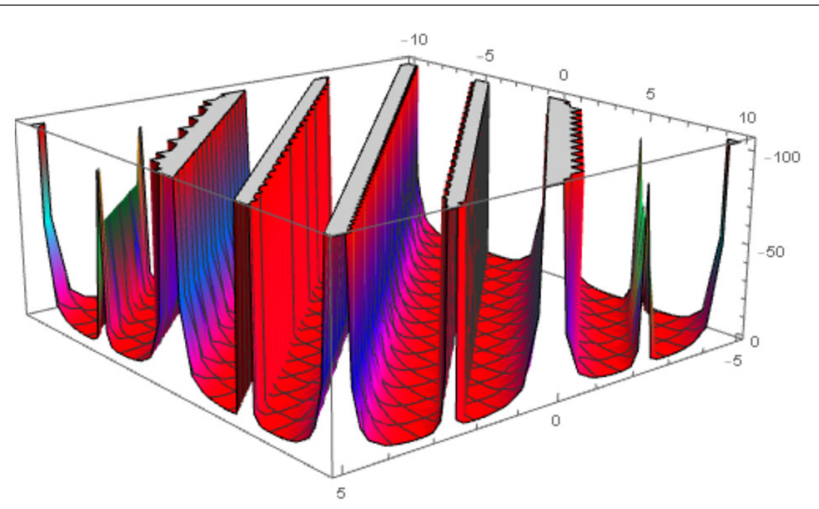

Figure 8 Periodic wave solution of $u_{12}(\eta)$ for $\alpha=0.9, a_{1}=11, \lambda=-100, b_{0}=1, V=10$

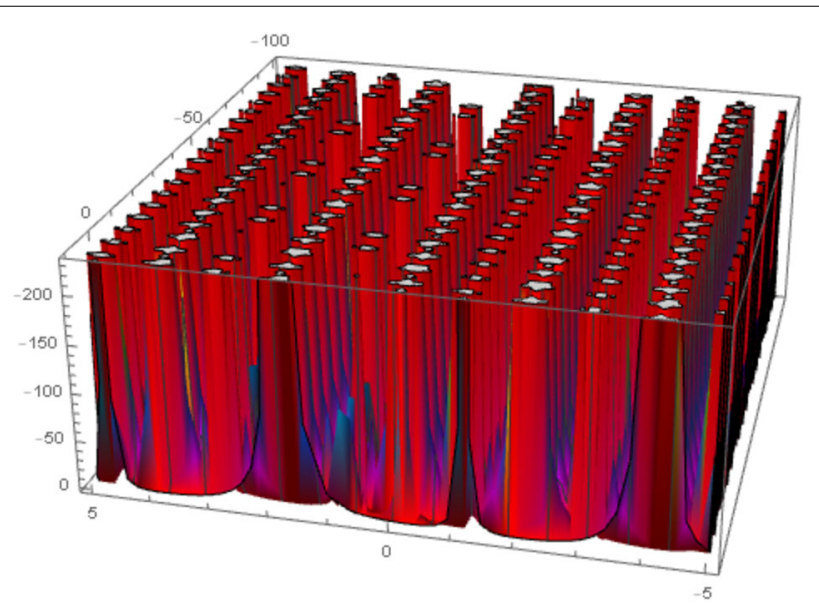

Figure 9 Periodic wave solution of $u_{19}(\eta)$ for $\alpha=0.30, a_{1}=11, \lambda=0.77, b_{0}=1, V=10, b_{1}=100$

\section{Conclusions}

We have successfully employed the proposed technique to gain the generalized solitary solutions of the simplified modified Camassa-Holm (SMCH) equation in the article. The solitary solutions are periodic, trigonometric, hyperbolic, and rational functions. The 


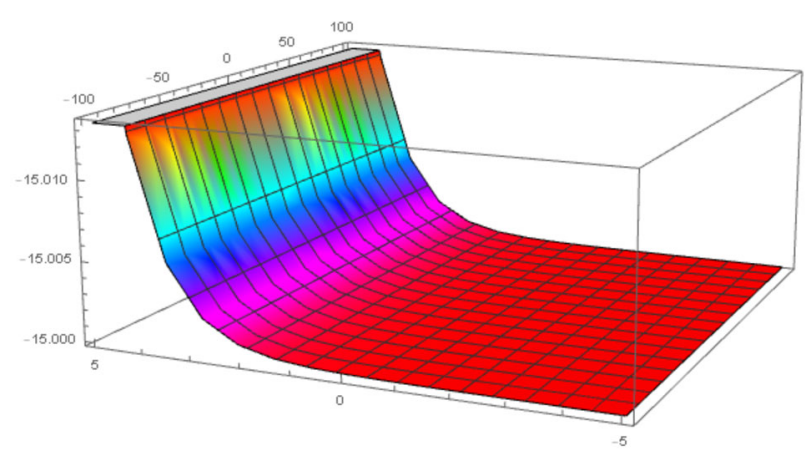

Figure 10 Solitonic solution of $u_{16}(\eta)$ for $\alpha=0.25, a_{0}=0.5, \lambda=-1, b_{0}=2, V=0.1, k=3$

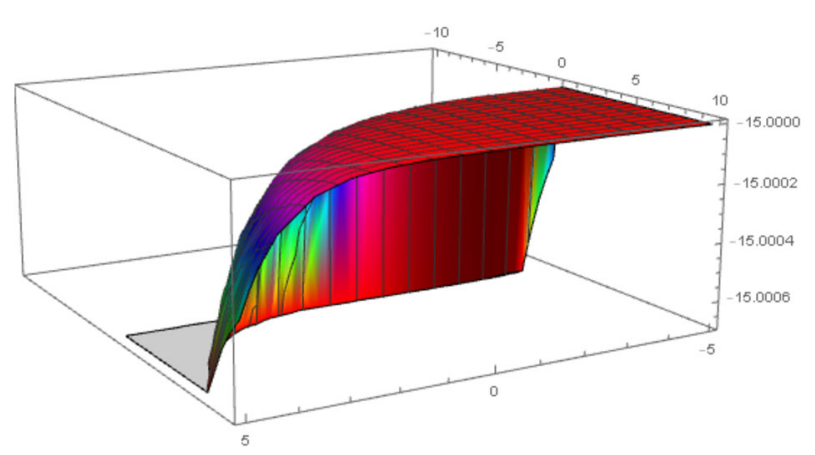

Figure 11 Solitonic solution of $u_{14}(\eta)$ for $\alpha=0.9, a_{0}=0.5, \lambda=-1, b_{0}=11, V=0.1, k=3$

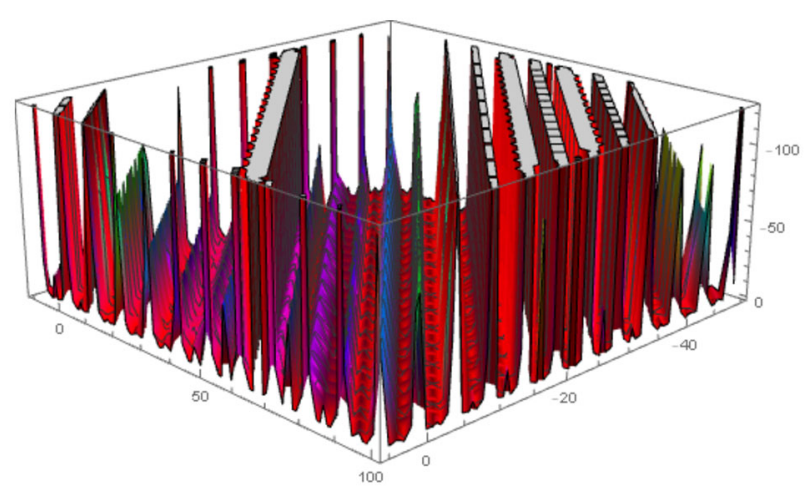

Figure 12 Periodic wave solution of $u_{15}(\eta)$ for $\alpha=0.1, a_{11}=11, \lambda=3, b_{0}=1, V=12, b_{1}=3$

gained results may express a variety of new features of waves. By comparing our results, we concluded that some of the results are similar to the current literature, while the others are newly discovered, and were not explored elsewhere. The method is reliable in handling to initiate new results and we selected a new class of exact solutions. It is investigated that the physical parameters significantly modify the wave dynamics. The solutions formed in this paper can be beneficial in the study of wave breaking. Wave breaking is used in atmospheric gravity waves and in plasma physics. It is also utilized in the analysis for discussing local well-posedness and global existence in non-peaked solutions. The suggested 


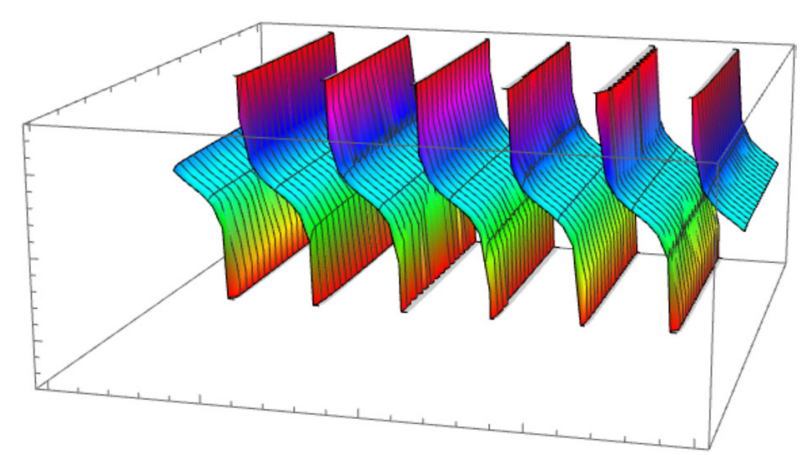

Figure 13 Solitonic solution of $u_{19}(\eta)$ for $\alpha=0.25, a_{1}=1, b_{1}=1, V=12, k=5$

procedure fully approved the dependability of our computational work and could be implemented to study other physical problems.

\section{Acknowledgements}

Not applicable.

Funding

None.

Availability of data and materials

None.

\section{Competing interests}

The authors declare that they have no competing interests.

\section{Authors' contributions}

All authors contributed to writing the draft and the software and all reviewed and approved the final version of the manuscript.

\section{Author details}

${ }^{1}$ Informetrics Research Group, Ton Duc Thang University, Ho Chi Minh City, Vietnam. ${ }^{2}$ Faculty of Mathematics \& Statistics, Ton Duc Thang University, Ho Chi Minh City, Vietnam. ${ }^{3}$ Department of Mathematics and Statistics, Faculty of Social Sciences, Institute of Southern Punjab, Multan, Pakistan. ${ }^{4}$ Department of Mathematics, Faculty of Sciences, Government, College University Faisalabad, Faisalabad, Pakistan. ${ }^{5}$ Centre for Advanced Studies in Pure and Applied Mathematics, Bahauddin Zakariya University, Multan, Pakistan. ${ }^{6}$ Department of Mathematics, Cankaya University, Ankara, Turkey. ${ }^{7}$ Institute of Space Sciences, Magurele-Bucharest, Romania. ${ }^{8}$ Department of Medical Research, China Medical University Hospital, China Medical University, Taichung, Taiwan. ${ }^{9}$ Department of Mathematics, College of Arts and Science, Prince Sattam bin Abdulaziz University, Wadi Aldawaser, Saudi Arabia.

\section{Publisher's Note}

Springer Nature remains neutral with regard to jurisdictional claims in published maps and institutional affiliations.

Received: 9 March 2020 Accepted: 3 June 2020 Published online: 22 June 2020

\section{References}

1. Ahmad, H., Seadawy, A.R., Khan, T.A., Thounthong, P.: Analytic approximate solutions for some nonlinear parabolic dynamical wave equations. J. Taibah Univ. Sci. 14, 346-358 (2020)

2. Seadawy, A.R., Lu, D., Yue, C.: Travelling wave solutions of the generalized nonlinear fifth-order KdV water wave equations and its stability. J. Taibah Univ. Sci. 11, 623-633 (2017)

3. Seadawy, A.R., Manafian, J.: New soliton solution to the longitudinal wave equation in a magneto-electro-elastic circular rod. Results Phys. 8, 1158-1167 (2018)

4. Hossain, A.K.S., Akbar, M.A., Azad, M.A.K.: The closed form solutions of simplified MCH equation and third extended fifth order nonlinear equation. Propuls. Power Res. 8, 163-172 (2019)

5. Ghanbari, B., Liu, J.G.: Exact solitary wave solutions to the $(2+1)$-dimensional generalised Camassa-Holm-Kadomtsev-Petviashvili (K-P) equation. Pramana 94, 21 (2020)

6. Ghanbari, B., Baleanu, D.: New solutions of Gardner's equation using two analytical methods. Front. Phys. 7, 202 (2019)

7. Belmor, S., Ravichandran, C., Jarad, F.: Nonlinear generalized fractional differential equations with generalized fractional integral conditions. J. Taibah Univ. Sci. 14, 114-123 (2020) 
8. Valliammal, N., Ravichandran, C., Hammouch, Z., Baskonus, H.M.: A new investigation on fractional-ordered neutral differential systems with state-dependent delay. Int. J. Nonlinear Sci. Numer. Simul. 20, 803-809 (2019)

9. Ravichandran, C., Valliammal, N., Nieto, J.J.: New results on exact controllability of a class of fractional neutral integro-differential systems with state-dependent delay in Banach spaces. J. Franklin Inst. 356, 1535-1565 (2019)

10. Jothimani, K., Kaliraj, K., Hammouch, Z., Ravichandran, C.: New results on controllability in the framework of fractional integrodifferential equations with nondense domain. Eur. Phys. J. Plus 134, 44 (2019)

11. Islam, M.N., Asaduzzaman, M., Ali, M.S.: Exact wave solutions to the simplified modified Camassa-Holm equation in mathematical physics. AIMS Math. 5, 26-41 (2019)

12. Khater, A.H., Callebaut, D.K., Seadawy, A.R.: General soliton solutions for nonlinear dispersive waves in convective type instabilities. Phys. Scr. 74, 384 (2006)

13. Seadawy, A.R.: Three-dimensional weakly nonlinear shallow water waves regime and its traveling wave solutions. Int. J. Comput. Methods 15, 1850017 (2018)

14. Khater, A.H., Callebaut, D.K., Malfliet, W., Seadawy, A.R.: Nonlinear dispersive Rayleigh-Taylor instabilities in magnetohydrodynamic flows. Phys. Scr. 64, 533 (2001)

15. Seadawy, A.R.: Solitary wave solutions of two-dimensional nonlinear Kadomtsev-Petviashvili dynamic equation in dust-acoustic plasmas. Pramana 89, 49 (2017)

16. Alqudah, M.A., Ravichandran, C., Abdeljawad, T., Valliammal, N.: New results on Caputo fractional-order neutral differential inclusions without compactness. Adv. Differ. Equ. 2019, 528 (2019)

17. Jafari, H., Tajadodi, H., Baleanu, D.: Application of a homogeneous balance method to exact solutions of nonlinear fractional evolution equations. J. Comput. Nonlinear Dyn. 9, 021019 (2014)

18. Bekir, A., Güner, Ö., Cevikel, A.C.: Fractional complex transform and exp-function methods for fractional differential equations. Abstr. Appl. Anal. 2013, Article ID 426462 (2013)

19. Rizvi, S.T.R., Ali, K., Bashir, S., Younis, M., Ashraf, R., Ahmad, M.O.: Exact soliton of (2+1)-dimensional fractional Schrödinger equation. Superlattices Microstruct. 107, 234-239 (2017)

20. Sezer, S.A., Yildırım, A., Mohyud-Din, S.T.: He's homotopy perturbation method for solving the fractional KdV-Burgers-Kuramoto equation. Int. J. Numer. Methods Heat Fluid Flow 21, 448-458 (2011)

21. Camassa, R., Holm, D.D.: An integrable shallow water equation with peaked solitons. Phys. Rev. Lett. 71, 1661 (1993)

22. Liu, X., Tian, L., Wu, Y.: Application of (G'/G)-expansion method to two nonlinear evolution equations. Appl. Math. Comput. 217, 1376-1384 (2010)

23. Najafi, M., Arbabi, S.: He's semi-inverse method for Camassa-Holm equation and simplified modified Camassa-Holm equation. Int. J. Phys. Res. 1, 1-6 (2013)

24. Abbasbandy, S.: Solitary wave solutions to the modified form of Camassa-Holm equation by means of the homotopy analysis method. Chaos Solitons Fractals 39, 428-435 (2009)

25. Lu, D., Seadawy, A.R., Iqbal, M.: Construction of new solitary wave solutions of generalized Zakharov-Kuznetsov-Benjamin-Bona-Mahony and simplified modified form of Camassa-Holm equations. Open Phys. 16, 896-909 (2018)

26. Alam, M.N., Akbar, M.A.: Some new exact traveling wave solutions to the simplified MCH equation and the (1 + 1)-dimensional combined KdV-mKdV equations. J. Assoc. Arab Univ. Basic Appl. Sci. 17, 6-13 (2015)

27. Li, C., Qian, D., Chen, Y.: On Riemann-Liouville and Caputo derivatives. Discrete Dyn. Nat. Soc. 2011, Article ID 562494 (2011)

28. Gündoğdu, H., Gözükızl, Ö.F:: On different kinds of solutions to simplified modified form of a Camassa-Holm equation. J. Appl. Math. Comput. Mech. 18, 31-40 (2019)

29. Mohyud-Din, S.T., Ali, A.: New exact solutions of time-fractional simplified Camassa Holm equation via rational Exp $\phi(\eta)$-expansion method. J. Comput. Theor. Nanosci. 14, 870-878 (2017)

30. Khater, A.H., Callebaut, D.K., Helal, M.A., Seadawy, A.R.: Variational method for the nonlinear dynamics of an elliptic magnetic stagnation line. Eur. Phys. J., D, At. Mol. Opt. Phys. 39, 237-245 (2006)

\section{Submit your manuscript to a SpringerOpen ${ }^{\circ}$ journal and benefit from:}

- Convenient online submission

- Rigorous peer review

- Open access: articles freely available online

- High visibility within the field

- Retaining the copyright to your article

Submit your next manuscript at $\gg$ springeropen.com 